\title{
La importancia de la profesionalización del servicio público. avances y retrocesos en materia de profesionalización en el estado de nuevo león
}

The importance of professionalization of the public service. advances and setbacks in professionalization in the state of nuevo león.

\author{
Xunaxhi Monserrat, Pineda-Rasgado ${ }^{1}$ \\ Universidad Autónoma del Estado de México
}

\begin{abstract}
RESUMEN
El presente artículo es producto de una revisión bibliográfica, cuyo objetivo consistió en mostrar evidencia de la importancia de la profesionalización del servicio público en el Estado de Nuevo León. Se aplicó el método de análisis, con un enfoque cualitativo, diseño no experimental, bajo un nivel documental-bibliográfico transversal. Tras la revisión documental se encontró que: Por años, distintos países han realizado esfuerzos por consolidar un sistema de profesionalización que aseguren la carrera dentro del sector público a las personas más capaces. En México, por ejemplo, se cuenta con una ley que tiene aplicación a nivel federal para el Sistema Profesional de Carrera, sin embargo, en el presente estudio se intenta brindar un panorama general de las circunstancias en que este sistema de profesionalización se encuentra en las entidades federativas y de manera específica en el Estado de Nuevo León, primer gobierno estatal independiente.
\end{abstract}

Palabras clave: Desparadojización, Equivalencia funcional, observación de segundo orden, método funcional.

\begin{abstract}
This article is the product of a literature review, whose aim consisted in showing evidence of the importance of the public service professionalization in the state of Nuevo León. The method of analysis was applied with a qualitative approach, non-experimental design, under a documentary-literature cross sectional level. After the documentary review, it was found that: efforts have been made by different countries for years in order to consolidate a professionalization system that assures the career within the public sector to the most capable people. In Mexico, for example, there is an applicable law at a federal level for the Career Professional System, however, in this study, it is intended to give an overall picture of the circumstances of this professionalization system is in the federal entities and in a specific way in the state of Nuevo León, first independent state government.
\end{abstract}

Key words: De-Paradoxofication, Fuctional Equivalent, Second-order Observation, Fuctional Method.

Cómo referenciar este artículo: Pineda-Rasgado, X. M. (2017). La importancia de la profesionalización del servicio público. avances y retrocesos en materia de profesionalización en el estado de nuevo león. Política, Globalidad y Ciudadanía, 41-53.

Recibido: 30 de Julio 2016 - Aceptado: 15 de Septiembre 2016

(oc) BY-NC-ND

1 Maestrante en Ciencias Políticas, Universidad Autónoma de Nuevo León. Email: xmpinedar@gmail.com

Revista Política, Globalidad y Ciudadanía, Vol. 3 No. 5, Enero - Junio 2017, Universidad Autónoma de Nuevo León, Monterrey, México Monterrey, México, ISSN 2395-8448. pp 44-56. http://revpoliticas.uanl.mx/index.php/RPGyC/article/view/63 


\section{1- INTRODUCCIÓN}

El desarrollo constante de las sociedades vuelve cada vez más complejas las responsabilidades del Estado. Hoy por hoy, pareciera inconcebible que las personas responsables de aspectos como la seguridad, la economía, las finanzas, la educación, entre otras, carezcan de algún tipo de especialización en la materia. En el entendido de que el gobierno, a través de la administración pública debe procurar el bienestar social, resulta indispensable la existencia de un cuerpo de funcionarios del estado que se encarguen no solo de representar el poder público, sino que con base en leyes y ordenamientos administrativos ejerzan dicho poder a fin de satisfacer de la mejor manera las necesidades públicas.

La naturaleza misma de las tareas del Estado exige que las personas que encabecen la administración pública cuenten con cierta preparación, conocimientos, habilidades y aptitudes específicas que les permita desempeñar de manera satisfactoria su labor. Es entonces, bajo la premisa anterior que destaca la importancia del estudio de la implementación del Servicio Civil de Carrera dentro del sector público.

Ahora bien, el interés por los sistemas de carrera dentro de las administraciones públicas no es un tema nuevo. En México, por ejemplo, existe desde el año 2003 una Ley del Servicio Profesional de Carrera con la cual se busca establecer un sistema de profesionalización en la administración pública federal que pudiera garantizar la integración de un cuerpo de funcionarios y servidores públicos preparados y capaces para el desempeño de la función pública. Sin embargo, esta es una política pública que sigue siendo cuestionable debido a que no se percibe como que el sistema de profesionalización funcione de la manera esperada.

Estudios como el de Sánchez (2017) comprueban lo anterior, pues a través del análisis de datos proporcionados por la Secretaría de la Función Pública (SFP) a través de una solicitud de acceso a la información pública, le es posible señalar que el sistema de carrera no está operando de manera correcta, pues ni siquiera se está cumpliendo con la Ley que rige el SPC. Los datos analizados por Sánchez muestran que el número de puestos ocupados con titulares y eventuales son mucho mayores a los puestos reportados como sujetos al SPC, así mismo señala que los servidores públicos no están cumpliendo con la capacitación que la ley señala como obligatoria. En ese contexto, surge el interés por estudiar la situación en la que se encuentran los gobiernos locales en materia de profesionalización, y de manera específica el gobierno de Nuevo León.

Con base en el Manual de Administración Pública que emite la Organización de las Naciones Unidas (ONU), Pardo (2005b) señala que para la implementación de un Servicio Civil de Carrera deben existir dos elementos básicos: una ley de administración del personal público así como un organismo responsable de su aplicación. En ese sentido, el presente estudio resulta ser de carácter exploratorio, pues busca ofrecer un acercamiento al conocimiento de las recientes condiciones del gobierno estatal en materia de profesionalización de la función pública. El objetivo es indagar en la legislación existente a fin de poder explicar cuál es el marco jurídico que actualmente rige la profesionalización en el gobierno estatal, si existe un Servicio Civil de Carrera que coadyuve la gestión de los recursos humanos dentro del aparato administrativo, cuál o cuáles son los organismos que operan dicho sistema y de qué manera funciona.

En la primera parte del documento se busca definir y diferenciar los conceptos sobre los que versa esta investigación, el Servicio Civil de Carrera y la Profesionalización. Posteriormente, se busca resaltar la importancia que guarda la existencia de los sistemas de carrera para frenar prácticas antiéticas dentro del servicio público. En el penúltimo apartado se explica cuáles son los esfuerzos reales que han existido por parte del gobierno federal y algunos gobiernos locales por instaurar un Servicio Profesional de Carrera. Finalmente, a la luz de la teoría y la experiencia federal, podrán señalarse los avances y retrocesos que ha mostrado el gobierno de Nuevo León en materia de profesionalización, así como la aparente ambigüedad que existe sobre la prevalencia de un Sistema de Carrera para la burocracia neoleonesa.

\section{2- FUNDAMENTO TEÓRICO}

\section{Servicio Civil de Carrera y Profesionalización}

México es un país que cotidianamente asume a su gobierno con poca capacidad para responder a las

Revista Política, Globalidad y Ciudadanía, Vol. 3 No. 5, Enero - Junio 2017, Universidad Autónoma de Nuevo León, Monterrey, México Monterrey, México, ISSN 2395-8448. pp 44-56. http://revpoliticas.uanl.mx/index.php/RPGyC/article/view/63 
necesidades de los ciudadanos. Incluso, el término burocracia se ha diluido y se ha alejado de lo que Weber (2001) entendía como el cuerpo de funcionarios del estado, responsables de representar y ejercer el poder público. Sin embargo, en la cultura popular, la burocracia se percibe como el grupo de personas e instituciones que entorpecen la resolución de problemas públicos. Buena parte de este ideario cultural respecto a la burocracia se debe en buena medida a la falta de profesionales estables dentro de los gobiernos y al uso faccioso de esta como recurso del poder político y de los intereses corporativos totalmente opuestos al modelo weberiano (Echebarría, 2008).

En la introducción de este documento se hizo mención de distintos conceptos que pudieran originar confusión pues pudieran parecer sinónimos, Servicio Civil de Carrera (SCC) y Profesionalización. De acuerdo con Pardo (2005b) el SCC es: "un conjunto de acciones sistemáticas mediante las cuales los servidores públicos pueden ingresar, permanecer y desarrollarse profesionalmente dentro de la administración pública, proporcionando a su vez niveles altos de eficiencia y eficacia que redunden en el cumplimiento óptimo de los objetivos institucionales como respuesta a las demandas públicas que el gobierno recibe por conducto de su aparato administrativo" (p. 9). Es decir, la autora describe netamente un sistema que permite gestionar de manera ordenada los recursos humanos.

Además, los miembros de este tipo de sistemas constituyen un cuerpo apolítico y permanente de funcionarios que forman la espina dorsal de una determinada administración. Los elementos esenciales de un sistema de carrera son una ley básica de administración de personal y un organismo responsable de su aplicación (Naciones Unidas, 1962). Los principios fundamentales dentro de los sistemas de carrera son: el mérito, y la igualdad de oportunidades.

El principio del mérito supone que los cargos públicos deben estar ocupados por las personas más capaces y preparadas, es decir, aquellos que "merecen" las designaciones gracias a sus conocimientos, habilidades y aptitudes (Dussauge, 2002). Por otra parte, la igualdad de oportunidades establece que el acceso a los cargos no puede estar condicionado por la situación económica, social o por el género de los aspirantes. La igualdad implica que cualquier persona interesada en un puesto de la estructura burocrática puede acceder a él sin discriminación alguna, con las únicas limitaciones de sus propias capacidades y por supuesto, las necesidades del cargo (Dussauge, 2002).

Vale la pena rescatar un tercer elemento, este es el referente a la estabilidad en el cargo. Esto significa que los funcionarios lleven a cabo sus responsabilidades sin suponer que los cargos son de su propiedad, pues ellos se encuentran sometidos a una rigurosa disciplina y vigilancia administrativa. Es por eso que los funcionarios deben estar en constante perfeccionamiento. La estabilidad del cargo es un mecanismo que permite evitar la existencia de un sistema de botín que ampare movimientos y despojos dentro de la administración (Pardo, 2005a).

Los rasgos característicos de los servicios de carrera son, principalmente, la selección mediante exámenes competitivos; las restricciones a la remoción arbitraria del cargo; la exigencia de neutralidad política a los funcionarios que aspiran a entrar y permanecer; y la existencia de un cuerpo regulador independiente que vigile el funcionamiento integral del sistema. El objetivo principal de estos sistemas, es conseguir la permanencia de los funcionarios más capaces a pesar de los cambios es la estructura política del gobierno (Pardo, 2005a). En otras palabras, lograr la estabilidad dentro del aparato administrativo pese a la inestabilidad política que pudiera existir.

Los sistemas de carrera en el servicio público no es una propuesta netamente reciente, como tampoco lo es la falta de confianza en las instituciones, la corrupción, el clientelismo, la falta de personal público especializado, etcétera, en los diferentes gobiernos. Pero todo esto último es lo que ha llevado a distintos países a tratar de implementar un Servicio de Carrera en sus gobiernos que sirva como instrumento para corregir y reivindicar la imagen de las administraciones públicas, mismas que en su mayoría se han convertido, frente a los ciudadanos, en un sinónimo de la ineficiencia gracias a la falta de capacidad de los servidores públicos para atender las necesidades sociales.

Muchos gobiernos alrededor del mundo, incluido México, reconocen que la adopción de un sistema como este permitiría a la función pública combatir el deficiente desempeño del servicio público, y brindará la capacidad necesaria a los servidores para cumplir con las tareas que les competen, así como poder

Revista Política, Globalidad y Ciudadanía, Vol. 3 No. 5, Enero - Junio 2017, Universidad Autónoma de Nuevo León, Monterrey, México Monterrey, México, ISSN 2395-8448. pp 44-56. http://revpoliticas.uanl.mx/index.php/RPGyC/article/view/63 
desarrollar mayores y mejores políticas públicas. Eso trae como resultado no solo la existencia de un buen gobierno, efectivo en respuesta y con servicios de calidad, sino que además combatirá de manera positiva la crisis de legitimidad que actualmente atraviesan.

Lo conceptualizado sobre el Servicio Civil en los párrafos anteriores puede provocar cierta confusión con la definición que retoma Longo (2008) sobre la profesionalización. Según el autor, la Carta Interamericana de la Función Pública define en su preámbulo que la profesionalización es "la garantía de posesión por los servidores públicos de una serie de atributos como el mérito, la capacidad, la vocación de servicio, la eficacia en el desempeño de su función, la responsabilidad, la honestidad y la adhesión a los principios y valores de la democracia" (p. 45).

Según explica Longo (2008), existe una relación entre la posesión de estos atributos con la existencia de un sistema de gestión del empleo. Es decir, que los principios de eficacia y eficiencia son compatibles con el mérito e imparcialidad de los sistemas de carrera. En otras palabras, la idea de profesionalización consiste en hacer más profesional al servicio civil. En este punto resulta pertinente recordar el paradigma de la Nueva Gestión Pública, pues es este nuevo enfoque de la administración pública el que de alguna manera origina y pone sobre la mesa la importancia de la profesionalización de las burocracias.

En las últimas décadas del siglo XX, surge en distintos países un nuevo modelo de gestión pública llamado Nueva Gerencia Pública (NGP). La idea principal de este modelo residía en obtener una mayor eficiencia en el trabajo gubernamental implementando en el quehacer público los métodos y prácticas que se aplican en las organizaciones del sector privado.

La llegada de este nuevo paradigma dentro de la administración, consistía en un modelo que va del énfasis en la política pública, a uno en las capacidades gerenciales; de un fuerte énfasis en los procesos, a un énfasis en los resultados; de jerarquías ordenadas, a la competencia en espacios de mercados, o cuasi mercados; de seguridad en el trabajo y salarios precisos para la burocracia, a evaluación del desempeño y firma de contratos específicos amarrados a la obtención de ciertos resultados (Arellano, 2002).

La Nueva Gerencia Pública surge como respuesta a los problemas que presenta la Administración Pública Tradicional. Según Hood y Hughes (citados en Martínez, 2003b), la Administración Pública Tradicional presentaba dos grandes problemas. En primer lugar, la propia eficiencia del modelo burocrático se veía afectada por el excesivo celo respecto a las reglas y los procedimientos.

En segundo lugar, la siempre falaz separación entre políticos y administrativos, la pretendida despolitización de la burocracia, la deshumanización de los procesos racionalizadores o las propias condiciones de trabajo que imponía el modelo generaban dificultades en la tarea de control políticos (Martínez, 2003b)

En ese sentido, el modelo burocrático tradicional pasa a un modelo postburocrático que trae consigo una perspectiva diferente. En primer lugar, se pasa de enfocar las necesidades y perspectivas propias a enfocar las del cliente o ciudadano; Segundo, el modelo burocrático se define asimismo tanto por la cantidad de recursos que éste controla como por las áreas que éste desempeña, mientras que el postburocrático se define asimismo por los resultados que éste alcanza para sus clientes; Tercero, el burocrático se enclava en la rutina, el potsburocrático modifica sus operaciones en respuesta a las cambiantes demandas de sus servicios (Martínez, 2003b)

Cuarto, el burocrático insiste en seguir sus procedimientos estandarizados, el postburocrático se construye de la elección al interior de sus sistemas de operación atendiendo a un propósito; y quinto, el burocrático establece planes y políticas, el postburocrático engrana una comunicación de dos vías con sus clientes para revisar y evaluar la estrategia de sus operaciones (Martínez, 2003b).

A partir de estos elementos, la profesionalización se convierte en una herramienta fundamental de la NGP para trata de superar algunos de los problemas típicos de las administraciones tradicionales, tales como que, al permanecer estas sin vigilancia, se vuelven corruptas e incompetentes, además que se desarrolla dentro de ellas una burocracia más poderosa y prácticamente inamovible. El objetivo es convertir a las burocracias tradicionales en un cuerpo de funcionarios y servidores públicos altamente capacitados para atender de manera más eficiente las demandas ciudadanas.

La periódica evaluación del desempeño que se propone dentro del modelo profesional intenta acabar con esas prácticas. Primeramente, reduciendo el cuerpo de funcionarios al servicio del Estado, tratando de

Revista Política, Globalidad y Ciudadanía, Vol. 3 No. 5, Enero - Junio 2017, Universidad Autónoma de Nuevo León, Monterrey, México Monterrey, México, ISSN 2395-8448. pp 44-56. http://revpoliticas.uanl.mx/index.php/RPGyC/article/view/63 
establecer un sistema que no deje de lado la continuidad en el servicio pero que también permita contar con una nueva burocracia capaz y profesional.

\section{Clientelismo político: Ausencia de ética pública}

Además de la búsqueda de la eficiencia y la eficacia en el servicio público, la profesionalización es también el mecanismo que busca terminar con lo que, en Estados Unidos, durante el siglo XIX, se conoció como spoil system, en español conocido como clientelismo político o sistema de botín (Pardo, 1995).

Resulta lógico entender que el clientelismo político es la antítesis de los sistemas de carrera, el llamado "amiguismo" o "compadrazgo", son expresiones que permiten entender de una manera burda el significado del clientelismo político, que no es otra cosa más que la práctica de nombrar a ciertas personas para detentar cargos dentro de la burocracia por motivo de lealtad a cierta persona en el poder o a un partido político. Desafortunadamente, el nepotismo es algo que se da con mucha frecuencia en los gobiernos y lo que deja en evidencia es la ausencia de una formación ética del servicio público.

Por otra parte, el servicio de carrera tiene como principal eje de función, el mérito. Y por qué no decirlo, también la lealtad, pero no a cierta persona, sino a la administración como tal. El ingreso al servicio, así como la promoción dentro del mismo se fundamentan en un sistema meritocrático, completamente contrario a lo que sucede en las prácticas clientelares. El sistema clientelar se conforma por grupos de personas que giran en torno a un poderoso patrón que reparte los cargos como método de ingreso al servicio. Este patrón jerárquicamente puede fungir como presidente, secretarios de estado, gobernadores o alcaldes, según sea el caso (Zapata, 2016)

Estos valorarán con base en su propio beneficio y conveniencia a aquellos que habrán de ocupar los cargos públicos. En este sistema de botín, el requisito principal para ser seleccionado es la absoluta lealtad que demuestren a la persona que los eligió, dejando prácticamente en último término cuan preparados y aptos se encuentran para ejercer dichos cargos. En este sentido, el objetivo principal de esta burocracia clientelar consiste en cumplir única y exclusivamente con las tareas que les haya establecido el poderoso que los eligió, siendo éste el único al cual importe entregar cuentas, ya que ello permitirá obtener como recompensa el aseguramiento del puesto o un ascenso, según sea el caso (Hernández, 2000).

De modo que aquel funcionario que no forme parte de esta dinámica en la que la prioridad es satisfacer los intereses de quien esté al mando, puede ser removido de su cargo sin tomar en cuenta su trayectoria o si aportó algún beneficio para la institución o para la sociedad. De cierta manera este sistema también podría considerarse finito y a la vez cíclico, ya que cada clientela con su respectivo patrón disfruta de un tiempo determinado de labores para que al término de su administración ésta sea sustituida para dar paso a la siguiente clientela, la cual habrá de regirse bajo los mismos esquemas y principios.

Como resultado se obtiene una interminable lista de funcionarios que además de no estar profesionalizados, enfrentan una enorme inestabilidad laboral que usualmente desemboca en la inexistente continuidad de las políticas y acciones del gobierno anterior. Todo esto ha ocasionado daños profundos a las administraciones públicas, ya que este problema ha tenido tanta presencia en los gobiernos que el mismo sistema administrativo de alguna manera ha aprendido, heredado y continúa reproduciendo estas viciadas prácticas.

Son estas acciones las que han deteriorado la imagen del gobierno, y lo han convertido ante los ojos de la sociedad en un ente que carece de credibilidad y de capacidad de gestión de las tareas estatales. Una burocracia conformada por elementos sin ningún tipo de aptitud para el servicio público, da como resultado un baja calidad en los servicios públicos, carencia de política pública y la casi inexistente capacidad de respuesta de los gobiernos a las necesidades sociales. En esa lógica, el servicio de carrera debe buscar remediar ese problema permitiendo la existencia de un gobierno eficaz que goce de una continuidad en sus quehaceres sin importar la eventual renovación de aquellos ocupan los cargos superiores (Pineda, 2017).

Un servicio público no profesionalizado se convierte en un freno para el desarrollo de las administraciones, impide el establecimiento de filtros que aseguren la especialización y capacitación de los servidores y que a la vez se resistan a la instrumentación de mecanismos de mejora. Es por ello por lo que resulta imprescindible tomar medidas y llevar a cabo acciones que permitan la creación y el fortalecimiento de me-

Revista Política, Globalidad y Ciudadanía, Vol. 3 No. 5, Enero - Junio 2017, Universidad Autónoma de Nuevo León, Monterrey,

México Monterrey, México, ISSN 2395-8448. pp 44-56. http://revpoliticas.uanl.mx/index.php/RPGyC/article/view/63 
canismos que aseguren un servicio público integrado por personas aptas y capaces para gestionar las tareas que les sean encomendadas, y que su lealtad esté fundamentada en el objetivo de atender las necesidades sociales.

\section{Servicio Profesional de Carrera en México}

Tal y como se señaló con anterioridad, el esquema de la nueva gerencia pública se asienta sobre la idea de construir un modelo diferente de gobierno que funcione bajo preceptos empresariales, es decir que, tomando como ejemplo las prácticas de trabajo del sector privado se pretendía lograr un gobierno más eficiente y eficaz a través de la profesionalización del servicio público. En este contexto México comienza a tomar medidas orientadas a este nuevo enfoque a finales de la década de los ochenta, sin embargo, en tema de profesionalización, no es hasta el año 2003 que el interés genuino por profesionalizar al servicio púbico toma lugar.

Previamente se hizo la distinción entre Servicio Civil y profesionalización, se dijo que ambos conceptos son complementarios en horas no solo de asegurar un sistema que tenga como base fundamental el mérito, sino que también posea una esencia de la búsqueda de la eficiencia y eficacia en el servicio, trascendiendo la visión burocrática del cumplimiento exclusivo de la norma, a la búsqueda de resultados.

En ese tenor, el gobierno federal mexicano, busca consagrar esa complementación de conceptos y en el año 2003 publica en el diario de la federación la Ley del Servicio Profesional de la Administración Pública Federal. Esta ley tenía por objeto establecer las bases para la organización, funcionamiento y desarrollo del Sistema de Servicio Profesional de Carrera en las dependencias de la Administración Pública Federal Centralizada.

La ley se piensa también como una herramienta de gestión del capital humano que permitiría al gobierno federal organizar de manera adecuada al personal que acceda al sector público garantizando el ingreso, el desarrollo y la permanencia de servidores mediante el mérito y la evaluación permanente del desempeño. Al respecto, Martínez Puón (2003a), señala que esta ley más que de carácter laboral, es de carácter administrativo porque lo que crea es un régimen especial para los servidores públicos denominados "de confianza".

De manera clara, la ley señala a la Secretaría de la Función Pública (SFP) como el órgano encargado de dirigir, coordinar y evaluar el funcionamiento del sistema en las dependencias de la administración central y vigilar que se cumpla con los principios rectores de la norma. No obstante, cabe señalar que la operación del sistema quedará a cargo de cada una de las dependencias de la administración federal (Artículo $2^{\circ}$ ).

Las facultades que se otorgan a la SFP para cumplir su labor se señalan en su artículo $69^{\circ}$ de la ley, entre las que se rescatan: emitir los criterios y establecer los programas del sistema, elaborar el presupuesto anual para la operación del mismo, administrar los bienes y recursos, expedir los manuales de organización y procedimientos, dar seguimiento a la implantación y operación del sistema en cada dependencia y en caso de ser necesario, dictar las medidas correctivas que se requieran.

De igual manera, debe encargarse de aprobar los mecanismos y criterios de evaluación, promover y aprobar los programas de capacitación y actualización, establecer mecanismos necesarios para conocer la opinión ciudadana respecto al funcionamiento del sistema, y revisar de manera periódica la operación del Sistema en las diversas dependencias.

\section{Profesionalización en las Entidades Federativas}

En 2007, el Instituto Nacional para el Federalismo y el Desarrollo Municipal (INAFED) realizó un estudio diagnóstico sobre los sistemas de profesionalización existentes en 21 de las 32 entidades federativas del país. El objetivo del estudio consistía en valorar en términos de consistencia y funcionalidad las tendencias organizativas, de planeación y de los procesos de los sistemas de profesionalización que operan en distintas entidades federativas disponer de elementos de análisis para la formulación del Modelo de Sistema de Profesionalización tipo para servidores públicos de las administraciones estatales (INAFED, 2007). El reporte del INAFED ofrece una valoración general sobre las condiciones del sistema de profesionalización

Revista Política, Globalidad y Ciudadanía, Vol. 3 No. 5, Enero - Junio 2017, Universidad Autónoma de Nuevo León, Monterrey, México Monterrey, México, ISSN 2395-8448. pp 44-56. http://revpoliticas.uanl.mx/index.php/RPGyC/article/view/63 
en términos normativos, organizacionales y de funcionamiento. El documento no se centra en el análisis específico de cada Estado, por lo que a partir de la información proporcionada por cada estado, se brinda un panorama general de las administraciones locales.

La publicación de la ley del servicio profesional a nivel federal fue un avance importante en la materia debido a que esto impulsó a distintas administraciones locales a realizar esfuerzos orientados a mejorar el sistema existente, $y$ en algunos casos, a construir uno que les permita construir una burocracia profesional y capaz, que contribuya de manera positiva al desempeño del gobierno y a la mejora continua (INAFED, 2007). Las particularidades que se registran en las entidades federativas respecto a la profesionalización son diversas. En algunos casos, entidades que al menos cuentan con una ley clara que regule el sistema, tal es el caso de Zacatecas ${ }^{2}$, o el entonces Distrito Federal ${ }^{3}$. Por otra parte, estados que simplemente no se tiene un marco normativo claro, y en algunos otros se logra observar cómo aún se encuentran en proceso de desarrollo, es decir, no existen aún los elementos suficientes que permitan evaluar su funcionamiento.

\section{Profesionalización del Servicio Público en el Estado de Nuevo León}

Tal y como se ha tratado de explicar a lo largo del texto, establecer un sistema de profesionalización requiere de varios elementos técnicos y de organización. Es necesario contar con una normativa clara que defina el objetivo del sistema, la manera en que debe operar, el órgano encargado de su ejecución, etcétera. No obstante, vale la pena partir del entendido que la profesionalización, tal y como lo estudia Arroyo (2010), es una política pública que en primera instancia debe ser un elemento que integre la agenda política, pues mucho depende el adecuado funcionamiento del sistema de la voluntad del poder político para conseguirlo.

El Plan Estatal de Desarrollo 2016-2021 cuenta con nueve capítulos, de los cuales se rescatan el tercero, Gobierno Eficaz y Transparente, y el octavo, Prioridades de Gobierno. En ambos apartados se señala la importancia y a necesidad de fortalecer la capacidad de acción del gobierno, hacer del aparato estatal un gobierno capaz de responder a las necesidades ciudadanas. De manera específica, el tema 2 del tercer capítulo, de manera clara señala la profesionalización del servicio público como algo imperativo de este nuevo gobierno.

Lo anterior sirve para confirmar lo que se mencionaba al principio de este texto, los gobiernos verdaderamente entienden que la profesionalización se ha vuelto en un imperativo de los gobiernos, como un mecanismo que puede ayudar a la lucha contra la corrupción que tanto ha permeado en las administraciones públicas. Abogar por la profesionalización del servicio público parece incluso un discurso indispensable dentro de las plataformas de gobierno, sin embargo, tal parece que los esfuerzos reales y tangibles no acompañan ese entendido.

El estado de Nuevo León cuenta con una Ley del Servicio Civil, publicada en el Periódico Oficial del estado el 26 de junio de 1948, reformada por última vez el 29 de diciembre de 2017, y tiene por objeto regular las relaciones de trabajo del gobierno del estado con sus servidores, además de ampliarse para los trabajadores de los ayuntamientos. Esta legislación protege al trabajador desde el momento en que toma posesión del empleo o el cargo para el que haya sido designado o desde que aparezca su nombre en nómina o lista de raya (INAP, 19999). En otras palabras, a nivel estatal, esta es la ley que rige la relación laboral de los trabajadores públicos con el gobierno, señala entre otras cosas, los derechos y obligaciones de los trabajadores, horas laborales, descansos, salarios, entre otras cosas. Es decir, no es una norma que contemple la existencia de un sistema público de carrera que regule el ingreso, la capacitación, la promoción y la separación del cargo ${ }^{4}$.

2 Periódico Oficial del Gobierno del Estado de Zacatecas. Ley del Servicio Profesional de Carrera del Estado y Municipios de Zacatecas. 10 de junio de 2003.

3 Gaceta Oficial del Distrito Federal. Ley del Servicio Público de Carrera de la Administración Pública del Distrito Federal. 13 de junio de 2000.

4 Ley del Servicio Civil del Estado de Nuevo León. Disponible en: http://www.honl.gob.mx/trabajo_legislativo/leyes/leyes/ley_del_servicio_civil_del_estado_de_nuevo_leon/

Revista Política, Globalidad y Ciudadanía, Vol. 3 No. 5, Enero - Junio 2017, Universidad Autónoma de Nuevo León, Monterrey, México Monterrey, México, ISSN 2395-8448. pp 44-56. http://revpoliticas.uanl.mx/index.php/RPGyC/article/view/63 
No obstante, de acuerdo con la investigación de Arroyo (2010), el estado de Nuevo León tuvo un importante avance en la materia, pues como parte del Plan Estatal de Desarrollo 2004 - 2009, así como del Programa Sectorial de Modernización Administrativa y Competitividad Gubernamental, el sistema de profesionalización se formaliza mediante el Decreto que establece el Estatuto de Profesionalización para el Servicio Público del Estado de Nuevo León, en el cual se define el marco jurídico básico que regula el Sistema de Profesionalización de la Administración Pública de Nuevo León.

Con el mismo decreto se creó el Instituto de Profesionalización para el Servicio Público del Estado de Nuevo León como órgano rector del sistema, disponiendo para el desarrollo e implementación de la política, de infraestructura administrativa, capital humano, recursos tecnológicos y materiales, así como de la asignación de presupuesto para administrar, operar y desarrollar el sistema de profesionalización y los subsistemas que lo integran (Artículo $29^{\circ}$ ).

Sin embargo, el problema principal para que este estatuto se cumpla es que debería estar vigente. De acuerdo con el mismo estatuto, en su artículo $42^{\circ}$ señala que le corresponde a la Oficialía Mayor de Gobierno administrar y operar el Subsistema de Ingreso; desarrollar y administrar el Catálogo General de Puestos de la Administración Pública Estatal, en atención a las necesidades de profesionalización y en coordinación con el Instituto de Profesionalización; así como coordinar, integrar, analizar, validar y registrar los perfiles de puestos.

En la reforma de 2016 a la Ley orgánica de la administración pública estatal establece una nueva estructura orgánica en la que se sustituye a dicha oficialía por una Coordinación Ejecutiva de la Administración Pública del Estado. Lo lógico hubiera sido reformar y actualizar esa información en el estatuto de profesionalización, sin embargo, no fue así. En el mejor de los casos, pudiera suponerse que en la práctica, el sistema de profesionalización operaría omitiendo ese detalle de forma en la normatividad, sin embargo tal suposición resulta imposible debido a que una vez revisada la estructura de gobierno mediante la información pública en el portal del gobierno estatal, el Instituto de Profesionalización no se contempla en ningún sitio web, ni forma parte de algún organigrama. Ese instituto no se contempla en el listado de dependencias públicas que se encuentra disponible en el portal del gobierno del estado 5 .

La misma reforma a la ley orgánica del año 2016, sustituye el texto del artículo 31 que Arroyo (2010, p. 17) había tomado para su investigación, en la cual se señalaba en su fracción VII "llevar a cabo programas y acciones... relativos a establecer la profesionalización del servicio público en el Estado". El texto actual de ese mismo artículo es el siguiente: "La Secretaría de Administración es la dependencia encargada de administrar los recursos humanos, materiales y servicios que requiera la Administración Pública del Estado", el mismo artículo 31 señala que a la Secretaría de Administración le atañe la responsabilidad de programar y celebrar los contratos por los cuales se realicen las contrataciones de recursos humanos, así como tramitar los nombramientos, promociones, cambios de adscripción, licencias, bajas y jubilaciones de los servidores públicos del Poder Ejecutivo.

De más está decir, que esta reforma de 2016 a la ley orgánica, no solo deja de lado al estatuto del servicio profesional y al instituto de profesionalización como elementos que rijan la integración del servicio público, sino que además dota de total discrecionalidad al poder ejecutivo para contratar y nombrar a funcionarios y servidores públicos de la manera que más le convenga. Sin mencionar que se minimiza de manera exponencial la importancia de la implementación de un sistema profesional en el gobierno estatal pues la gestión de los recursos humanos del ejecutivo estatal, solo quedaron bajo la responsabilidad de una Dirección General perteneciente a la Secretaría de Administración.

5 Dependencias del Gobierno Estatal. Consultar en: http://www.nl.gob.mx/dependencias

Revista Política, Globalidad y Ciudadanía, Vol. 3 No. 5, Enero - Junio 2017, Universidad Autónoma de Nuevo León, Monterrey, México Monterrey, México, ISSN 2395-8448. pp 44-56. http://revpoliticas.uanl.mx/index.php/RPGyC/article/view/63 


\section{3- MÉTODO}

\section{Diseño}

El enfoque investigativo de la presente investigación es cualitativo, de acuerdo con Hernández, Batista y Fernández (2014) “Utiliza la recolección y análisis de los datos para afinar las preguntas de investigación o revelar nuevas interrogantes en el proceso de interpretación” (p.7).

Alcanzando un diseño no experimental "Que se realiza sin la manipulación deliberada de variables y en los que sólo se observan los fenómenos en su ambiente natural para después analizarlos” (Hernández, Batista y Fernández, 2014, p. 149).

El alcance establecido es el exploratorio "emplean cuando el objetivo consiste en examinar un tema poco estudiado o novedoso” (Hernández, Batista y Fernández, 2014, p. 91).

\section{Instrumentos}

Para la construcción del marco teórico-conceptual de la importancia de la profesionalización del servicio público en Nuevo León, se consultaron un total de cuarenta y dos referencias bibliográficas utilizándose como instrumento las ideas, argumentos y proyectos que fueron interpretados desde una perspectiva analítica y crítica.

\section{Procedimiento}

Con relación a la comprensión del problema de la investigación se recopilan fuentes secundarias de documentos académicos. En el marco de referencia se definen los conceptos básicos relativos a la profesionalización del servicio público en Nuevo León. Una vez recopilada y analizada la información se construye el documento objeto de este trabajo. Por último, se realizan las recomendaciones y conclusiones conforme a los objetivos trazados (Lechuga, 2018, p. 196).

\section{CONCLUSIONES}

Estos argumentos son relevantes por varias razones, en primer lugar, exponen la amplitud de perspectiva que permite el MF y la observación de segundo orden, pero, por otro lado, son una muestra fehaciente de que las críticas en torno al supuesto "conservadurismo" de la teoría luhmanniana y el método funcional no tienen origen en la reflexión teórico-metodológica, sino en el prejuicio y el desconocimiento.

Como sostiene Torres (en Luhman, 1998: 17), en nuestro medio, es común el rechazo al trabajo de Niklas Luhmann, bajo la queja de que su teoría resulta demasiado compleja. Ello tiene efectos hondos en el pensamiento sociológico, pues, como señalamos, o se critica a Luhmann desde la falta de comprensión o, sencillamente, se descarta como explicación posible. Esta situación ilumina la necesidad de elaborar ejercicios de 'traducción', de clarificación de la teoría luhmanniana y los recursos que involucra, con el ánimo de entablar diálogos académicos que excluyan perspectivas políticas o morales.

El método funcional, como señalamos, consiste en relacionar varias causas que son funcionalmente equivalentes para producir el mismo efecto, e intenta identificar cuál es el criterio de selección de una causa para producir cierto efecto y con relación a qué función lo cumple. Partir del reconocimiento del carácter abstracto de las funciones y de los problemas de referencia, observar la equivalencia entre las posibles soluciones del mismo, permite una mirada de gran angular, desontologiza, complejiza, transita por 'lugares' que, con el uso de otros métodos, quedan completamente invisibilizados.

En este sentido, reiteramos, a propósito del 'carácter conservador' atribuido al MF, que el funcionalismo no busca justificar algún tipo de efecto y después encontrar una causa que garantice la producción del mismo, más bien, utiliza la referencia a un tipo de efecto particular como estímulo para comparar y equiparar determinados hechos causales. Por ello, no procede a partir de la exclusión de todas las otras

Revista Política, Globalidad y Ciudadanía, Vol. 3 No. 5, Enero - Junio 2017, Universidad Autónoma de Nuevo León, Monterrey,

México Monterrey, México, ISSN 2395-8448. pp 44-56. http://revpoliticas.uanl.mx/index.php/RPGyC/article/view/63 
causas posibles y la designación de una, sino de la inclusión, pues su pretensión es identificar rendimientos equivalentes en la resolución de problemas particulares de los sistemas sociales.

Esta manera de proceder sólo es posible a partir de una construcción particular del concepto de observación, a saber, observar como el trazo de una distinción, como el ejercicio de sacar algo del unmarked space del mundo, como una operación que no adquiere sustancia alguna, sino que únicamente actualiza un lado de una forma, una forma que siempre aparece como 'invisible' para quien observa y que por ello reclama la perspectiva del segundo orden.

La observación de segundo orden permite identificar la distinción que utiliza el observador de primer orden, con ello es posible clarificar ¿qué es lo que el observador de primer orden excluye cuando utiliza esa distinción?, ¿qué es lo que ésta no le permite ver?, ¿por qué esa distinción y no otra? Dicha estrategia se traduce en una 'crítica' más profunda que cualquiera planteada por las 'teorías marxistas', en el sentido de que presenta una imagen compleja del mundo, que excluye explicaciones unilaterales y simplistas.

Mirar desde el segundo orden, permite acceder a un concepto de realidad que sólo puede ser resultado de una red de observaciones cuya 'consistencia' y validez se explica sólo a partir de su facticidad. Es decir, en la base del orden social no aparece ningún tipo de consenso o instrucción ontológica, sino un entramado de observaciones recursivas.

Pensar, desde la teoría de sistemas, a la sociedad moderna como resultado de una multiplicidad de observaciones permite reconocer que siempre existe 'el otro lado', que quien observa es incapaz de percibir las discontinuidades y las distinciones de las que parte la propia observación, es decir, es incapaz de ver lo que no ve, por tanto, es en este sentido que la observación de segundo orden se convierte en un recurso imprescindible en la sociología, pues permite ver lo que el observador de primer orden no puede.

Sólo en este contexto adquieren relevancia las posibles soluciones a los problemas, que no fueron seleccionadas. Bajo este concepto de realidad la noción de contingencia juega un papel central, pues la selección de una solución a un problema de referencia sólo es una entre otras soluciones posibles, es decir, es contingente.

A partir de ello es posible comprender, por un lado, la importancia de la relación entre el método funcional y la observación de segundo orden en la propia teoría luhmanniana y por otro, la relevancia de este binomio para el pensamiento sociológico, en el sentido de que, el MF, permite, también reconocer que su observación es una observación hecha por un observador, en este sentido, puede ser observada.

Finalmente consideramos importante enfatizar que la discusión desarrollada en el presente artículo refuta las críticas (prejuicios) señaladas al inicio de la exposición. Pues frente a la idea de que, por herencia parsoniana, el método funcional de Niklas Luhmann concibe al sistema en términos de orden y equilibrio, por tanto, las estructuras deben mantienen una función estática, es necesario recordar, que tal y como sostiene Galindo (2008: 58) en la reconstrucción que Luhmann elaboró del método funcional, buscó, fundamentalmente, rescatarlo de las premisas del estructural funcionalismo parsoniano.

Mientras que, para Parsons, la existencia del sistema social depende sólo de cuatro funciones, para Luhmann, como ya se demostró, la idea de función y estructura no permanece estática, por el contrario, éstos son vistos como componentes dinámicos que pueden ser transformados. El reconocimiento del dinamismo en el sistema, es decir, de que todo sistema social se compone de elementos basalmente inestables y que en este sentido las estructuras están supeditadas a las operaciones, responde a la segunda crítica, citada en las primeras líneas de este texto, que sostiene que el método funcional descuida el cambio y el 'conflicto'.

Por otro lado, la perspectiva formal del método funcional anula toda noción de actores portadores de funciones; como se especificó, para Niklas Luhmann la función no tiene sustancia, tampoco es un efecto a producir, ésta es únicamente un esquema lógico regulador, que organiza un ámbito de comparación de efectos equivalentes.

Reservamos el final de este artículo a la crítica según la cual el método funcional 'no cumple con los requerimientos mínimos que demanda la ciencia', porque consideramos sustancial, subrayar que pocas rutas de análisis permiten una imagen tan amplia de los problemas sociológicos, como la que logra el método funcional.

La posibilidad de observar las diversas opciones que pueden sustituirse en una relación de equivalencia

Revista Política, Globalidad y Ciudadanía, Vol. 3 No. 5, Enero - Junio 2017, Universidad Autónoma de Nuevo León, Monterrey,

México Monterrey, México, ISSN 2395-8448. pp 44-56. http://revpoliticas.uanl.mx/index.php/RPGyC/article/view/63 
y logran desparadojizar la paradoja del observador, el ejercicio de comparar diversas observaciones, científicas y cotidianas, la forma problema/ múltiples soluciones equivalentes del problema amplían la visión científica, como no lo había hecho antes ninguna perspectiva. La 'duda metódica' que se logra con la visión funcionalista es una innovación revolucionaria en sociología.

\section{REFERENCIAS}

Arnold, M. y Robles, F. 2000. Explorando caminos transilustrados más allá del neopositivismo. Cinta moebio 7: 49-66

Baecker, D. (2005). Form and forms of communication. Deutschland: Frankfurt am Main, editorial Suhrkamp.

Bateson, G. (1998). Pasos hacia una ecología de la mente, una aproximación revolucionaria a la autocomprensión del hombre. Lohlé-Lumen, Buenos Aires, Argentina.

Bednarz Jr, J. (1984). Functional Method and Phenomenology: the view of Niklas Luhmann. Human Studies, Vol 7, 343-362.

Bobbio, N. (1998). Diccionario de política, Vol 1. Siglo XXI editories.

Del Angel, J. S. (2008). La autorreproducción del sistema de la ciencia en el campo académico de la comunicación en México. Una reflexión a partir de Niklas Luhmann. Tesis de Doctorado, Universidad Veracruzana, Boca del Río, Veracruz, México.

Estrada, S. M. (2007). La comunidad armada rebelde y el EZLN: un estudio histórico y sociológico sobre las bases de apoyo zapatistas en las cañadas tojolabales de la Selva Lacandona, - 1a. ed. - México, D.F.: El Colegio de México, Centro de Estudios Sociológicos, 1930-2005. pp. 623.

Estrada, S. M. (2010). Consideraciones finales zapatismos locales. En Estrada S., M. y J.P., Viqueira (2010). Los indígenas de Chiapas y la rebelión zapatista: microhistorias políticas. México D.F.: El Colegio de México, pp. 419-434.

Estrada, S.; M. y Guerra B., E. (2012). Coda. La perspectiva sistémica para el estudio de los movimientos sociales: ¿sólo otro giro de tuerca? En Estrada S., M. (Coord.) Protesta social. Tres estudios sobre movimientos sociales en clave de la teoría de sistemas de Niklas Luhmann. México: D.f.. El Colegio de México.

Foucault, M. (2010). La arqueología del saber. 2 a ed., Siglo XXI, México.

Galindo M., J. (2008). Entre la necesidad y la contingencia: Autoobservación teórica de la sociología. Anthropos, Universidad Autónoma Metropolitana, México.

Günther, G. (1973). Life as Poly-Contexturality. En H. Fahrenbach (Hrsg.), Wirklichkeit und Reflexion, Festschrift für Walter Schulz, 187-210.

Günther, G. (1979). Cognition and Volition A Contribution to a Cybernetic Theory of Subjectivity. Beiträge zu einer opertionsfähigen Dialektik, Band 2, Felix Meier Verlag, Hamburg, pp. 203-240.

Habermas, J. (1990). Pensamento póst-metafísico. Estudos filosóficos/Jürgen Habermas. - Rio de Janeiro: Tempo Brasileiro, pp.271

Hernández, R., Fernández, C., y Baptista, P. (2014). Metodología de la investigación. México: Mc Graw Hill.

Laclau, E. (1995). The Time Is out of Joint. Diacritics, 25, 2, 85-96. 
Lechuga, J. (2018). Necesidades insatisfechas de una comunidad aledaña a una terminal portuaria de Barranquilla. Revista Pensamiento y Gestión, 45 (2) 181-210.

Luhmann, N. (1973). Función y Casualidad en "Ilustración sociológica y otros ensayos". Argentina: Buenos Aires, editorial Sur.

Luhmann, N. (2007). La sociedad de la sociedad (1a ed.). México: Herder, Universidad Iberoamericana, pp. 954.

Luhmann, N. (1992). Entrevista a Niklas Luhmann. En Torres N. Y Zermeño P., Estudios Sociológicos X: 30, 1992.

Luhmann, N. (1995). The Paradoxy of Observing Systems. Cultural Critique, No. 31, The Politics of Systems and Environments, Part II, pp. 37-55.

Luhmann, N. (1996). La ciencia de la sociedad. Universidad Iberoamericana /Editorial Anthropos, 1ed. en español.

Luhmann, N. (1998). Sistemas sociales, Lineamientos para una teoría general. Anthropos Editorial/ Universidad Iberoamericana/ CEJA, Pontifícia Universidad Javeriana, 582.

Luhmann, N. (1998,b). ¿Cómo se pueden observar estructuras latentes? En, Watlawick, Paul; Krieg Peters. El ojo del observador: contribuciones al constructivismo. Barcelona, Gedisa, 1998. pp 60-72.

Luhmann, N. (1999). Teoría de los sistemas sociales: Universidad Iberoamericana, artículos II, 1 ed. p. 233

Luhmann, N. (2006). Sociología del riesgo. Universidad Iberoamericana, México, D.F., p. 205

Luhmann, N. (2007, b). La religión de la sociedad, España, Madrid, 1a. edición en español, Editorial Trotta, $314 \mathrm{pp}$.

Luhmann, N. (2009). Introducción a la teoría de sistemas, lecciones publicadas por Javier Torres Nafarrate, Universidad iberoamericana, tercera reimpresión, México, D.F.

Luhmann, N. (2009, b). Sociología de la religión, Editorial Herder, 1a edición en español, México, D.F.

Marx, K. 1978. Contribución a la crítica a la economía política. Fondo de Cultura Económica.

Maturana R, H. (2009). La realidad: ¿objetiva o construida? II, fundamentos biológicos del conocimiento. Anthropos Editorial; México: UNiversidad Iberoamericana: Guadalajara (México): Insituto Tecnológico y de Estudios Superiores de Occidente (ITESO).

Maturana R, H. Y Varela G. F. (2003). De máquinas y seres vivos, autopoiésis: La organización de lo vivo. Buenos Aires, Lumen.

Maturana R, H. Y Varela G. F. (2003, b). El árbol del conocimiento: las bases biológicas del entendimiento humano. Buenos Aires: Lumen, p. 208.

Niels A, A. (2003). Discourse Analytic Strategies: Understanding; Foucault, Kosselleck, Laclau, Luhmann, UK, The policy press.

Pintos, J. L. (1999). Sociocibernética: Marco sistémico y esquema conceptual en Métodos y Técnicas cualitativas de Investigación. en Ciencias Sociales, Editorial Síntesis, Madrid.

Pintos, J. L. (2004). Revisión y ampliación de los elementos básicos de la Teoría de los Imaginarios Sociales. Publicado en SEMATA. Ciencias sociales y humanidades, 16, 17-52.

Rasch, W. (1997). Locating the Political: Schmitt, Mouffe, Luhmann, and the Possibility of Pluralism. International Review of Sociology, 7, 1, 103-114. 
Spencer B, G. (1979). Laws of form, New York, Dutton, a Division of Elsevier-Dutton Publishing Co., Inc., p.143

Stäheli, U. (2008). Figuras rivales del límite; dispersión, transgresión, antagonismo e indiferencia, en Laclau, aproximaciones críticas a su obra, Simon Critchley y Oliver Marchat (Compiladores), 1 Ed. Buenos Aires, Fondo de Cultura Económica, p. 444

Von Foerster, H. (1991). Las Semillas de la cibernetica. Colección: Terapia familiar, p. 224

Wall, D. (2005). Babylon and Beyond, The Economics of Anti-Capitalist, Anti-Globalist and Radical Green Movements. Chase Publishing Services Ltd, Fortescue, Sidmouth, EX10 9QG, England.

Zamorano F., R. (2008). Debate en torno a las concepciones del tiempo en sociología. Chile, Santiago, Cinta de Moebio, 31, 53-69. 